\title{
Rank Monotonicity in Centrality Measures
}

\author{
Paolo Boldi \\ Università degli Studi di Milano, Dipartimento di Informatica \\ Alessandro Luongo \\ Università degli Studi di Milano, Dipartimento di Informatica \\ Sebastiano Vigna* \\ Università degli Studi di Milano, Dipartimento di Informatica
}

\begin{abstract}
A measure of centrality is rank monotone if after adding an arc $x \rightarrow y$, all nodes with a score smaller than (or equal to) $y$ have still a score smaller than (or equal to) $y$. If in particular all nodes with a score smaller than or equal to $y$ get a score smaller than $y$ (i.e., all ties with $y$ are broken in favor of $y$ ) the measure is called strictly rank monotone. We prove that harmonic centrality is strictly rank monotone, whereas closeness is just rank monotone on strongly connected graphs, and that some other measures, including betweenness, are not rank monotone at all (sometimes not even on strongly connected graphs). Among spectral measures, damped scores such as Katz's index and PageRank are strictly rank monotone on all graphs, whereas the dominant eigenvector is strictly monotone on strongly connected graphs only.
\end{abstract}

\section{Introduction}

The study of centrality in networks goes back to the late forties. Since then, several measures of centrality with different properties have been published. This paper follows the line of (Boldi \& Vigna, 2014), which proposed axioms that characterize abstractly the behavior of centrality measures. The purpose of the study is to end up with detailed tables clarifying for each centrality measure whether it satisfies or not a certain axiom. This kind of knowledge makes it possible to understand the global behavior of the measure, reducing the need of dealing with anecdotal results.

The assessment of centrality measures in (Boldi \& Vigna, 2014) is based on simple guiding principles; a centrality measure should be robust (i.e., applicable to arbitrary directed graphs, possibly non-connected, without modifications) and understandable (it should have

* The authors have been supported by the Google Faculty Research Award "Entity disambiguation by geometric centralities". 
a clear combinatorial interpretation). In this paper we follow the same guidelines, but to allow for a more fine-grained granularity we observe also the behavior of centrality measures on strongly connected networks, for which sometimes results are quite different.

The main body of results of this paper concerns rank monotonicity. In (Boldi \& Vigna, 2014), the authors discuss score monotonicity - the property that when an $\operatorname{arc} x \rightarrow y$ is added to the graph, the score of $y$ strictly increases. As already remarked by Chien, Dwork, Kumar, Simon and Sivakumar (2004) in the case of PageRank, score monotonicity alone is not sufficient, as it just defines the change on $y$ : in principle, other nodes might change their score as well, in a preposterous way. They propose a rank monotonicity axiom, which we adopt in this paper, which states that after adding an $\operatorname{arc} x \rightarrow y$, all nodes with a score smaller than (or equal to) $y$ have still a score smaller than (or equal to) $y$. In intuitive terms, the rank of $y$ remains the same, or improves. In this paper we study also a strict variant, where we require that all nodes with a score smaller than or equal to $y$ get a score strictly smaller than $y$ (i.e., all ties with $y$ are broken in favor of $y$ ).

There are two main reasons to study a strict version: the first one is that the constant score is rank monotone, which shows that the non-strict axiom is quite weak; the second reason is that the strict version is very natural: if you have two persons $P$ and $Q$ on Twitter with the same centrality, adding a follower just to $P$ should make it more important than $Q$. Indeed, we will see that we can improve the result of rank monotonicity of PageRank proved in (Chien et al., 2004) to strict rank monotonicity, and under much milder hypotheses.

In any case, score and rank monotonicity complement themselves. Score monotonicity tells us that "something good happens". Rank monotonicity that "nothing bad happens". Strict rank monotonicity is a glorified combination of these two properties.

Our results suggest once again that simple measures based on distances, and in particular harmonic centrality, behave more predictably than some of the most sophisticated indices proposed in the literature.

\section{Related work}

Centrality is a central notion in the study of social networks: the first attempts to define centrality indices date back to the late 1940s and were attempted by the Group Networks Laboratory at MIT directed by Alex Bavelas (1948), in the framework of communication patterns and group collaboration (Leavitt, 1951; Bavelas et al., 1951). In the following decades, various measures of centrality were employed in many different contexts; see, for instance, (Cohn \& Marriott, 1958; Pitts, 1965; Beauchamp, 1965; Mackenzie, 1966).

The most classical notions of centrality are closeness centrality (Bavelas, 1948), and its variant proposed by Lin (1976), betweenness, introduced by Anthonisse (1971) under the name of rush and rediscovered later by Freeman (1977), Katz's index (Katz, 1953) and harmonic centrality, a variation of closeness based on the harmonic mean. ${ }^{1}$ While these notions of centrality are combinatorial in nature and based on the discrete structure of the network, another line of research studies spectral techniques (in the sense of linear algebra) to define centrality. The most well-known among the spectral centrality

1 Harmonic centrality appeared in a number of different context independently; some of its history is tracked in (Boldi \& Vigna, 2014). 
indices is the dominant eigenvector, introduced by Wei (1952) to rank sport teams and later generalized by Berge (1958) to arbitrary graphs, Seeley's index (Seeley, 1949), the stationary state of the natural Markov chain on the graph, the dominant singular vectors, introduced by Bonacich (1991) to rank simultaneously persons and groups and rediscovered later by Kleinberg (Kleinberg, 1999) as part of HITS, PageRank (Page et al., 1998) and SALSA (Lempel \& Moran, 2001).

Although centrality is certainly an important structural property of social networks, there is no consensus on what centrality is exactly or on its conceptual foundation. As Freeman observed, "several measures are often only vaguely related to the intuitive ideas they purport to index, and many are so complex that it is difficult or impossible to discover what, if anything, they are measuring" (Freeman, 1979).

The idea of formalizing the behavior of centrality indices through axioms was first attempted by Sabidussi, in his much-quoted paper on centrality (Sabidussi, 1966), where he discussed a set of axioms that should be satisfied by a sensible centrality measure on an undirected graph. A few years later, Nieminen (1973) attempted a similar formalization for directed graphs. More recently, Boldi and Vigna (2014) tried to propose a set of axioms capturing various interesting properties of centrality measures, and put a large spectrum of indices under test to see which ones satisfied them.

Some axiomatization in the literature are hard, in the sense that they lead to the definition of a unique measure satisfying the axioms. This is case with Seeley's index (Altman \& Tennenholtz, 2008) ${ }^{2}$, the dominant eigenvector and singular vector (Kitti, 2016), harmonic centrality (Garg, 2009), Katz's index and the dominant eigenvector (Dequiedt \& Zenou, 2014), and Pinski and Narin's (1976) bibliometric ranking (Palacios-Huerta \& Volij, 2004). The disadvantage of hard axiomatizations is that they usually require very specifically tailored axioms, which have little meaning (or cannot even be formulated) for a generic notion of centrality.

In this work, we ideally continue along the line started by Boldi and Vigna (2014), and take rank monotonicity into account. In their paper, Boldi and Vigna already proposed a notion of score monotonicity: a centrality measure is score monotone if ${ }^{3}$ adding a new arc $x \rightarrow y$ increases the centrality score of $y$. Score monotonicity, unfortunately, does not say much about the relative ranks of nodes after the addition of the arc: this is why we are interested in introducing a form of monotonicity of ranks. In the work of Chien, Dwork, Kumar, Simon and Sivakumar (2004), albeit targeted exclusively at PageRank, the authors provide a definition of rank monotonicity that we think captures the essence of the problem: when we add a new $\operatorname{arc} x \rightarrow y$, nodes with a score smaller than $y$ must continue to have a score smaller than that of $y$, while nodes with a score equal to $y$ must get a score that is

2 Note that the authors claim to axiomatize PageRank, but actually the definition they give lacks the "teleportation factor" $\alpha$.

3 This definition resembles an axiom proposed by Sabidussi (1966), but he additionally requires that all the other nodes do not decrease their score (a very stringent requirement indeed, that cannot be satisfied by several measures; most notably, by most indices that are normalized). 
smaller than or equal to that of $y .{ }^{4}$ This notion can be seen as a generalization of the wellknown rank-monotonicity principle used in the theory of social choice, as in (Fishburn, 1982).

Very recently, Brandes, Kosub and Nick (2012) proposed to call radial a centrality in which the addition of an arc $x \rightarrow y$ does not decrease the rank of $y$, in the sense that when we add an arc towards $y$, nodes with a score smaller than or equal to $y$ continue to have this property. This is actually a weaker condition than the one used in (Chien et al., 2004) to state rank monotonicity, as it makes a form of non-monotonicity of ranks possible: nodes with a score smaller than $y$ might end up having a score equal to $y$ when we add an arc towards $y$.

\section{Definitions and conventions}

In this paper, we consider directed graphs defined by a set $N$ of $n$ nodes and a set $A \subseteq N \times N$ of arcs; we write $x \rightarrow y$ when $\langle x, y\rangle \in A$ and call $x$ and $y$ the source and target of the arc, respectively. An arc with the same source and target is called a loop.

The transpose of a graph is obtained by reversing all arc directions (i.e., it has an arc $y \rightarrow x$ for every arc $x \rightarrow y$ of the original graph). A symmetric graph is a graph such that $x \rightarrow y$ whenever $y \rightarrow x$; such a graph is fixed by transposition, and can be identified with an undirected graph, that is, a graph whose arcs (usually called edges) are subsets of one or two nodes. A successor of $x$ is a node $y$ such that $x \rightarrow y$, and a predecessor of $x$ is a node $y$ such that $y \rightarrow x$. The outdegree $d^{+}(x)$ of a node $x$ is the number of its successors, and the indegree $d^{-}(x)$ is the number of its predecessors.

A path (of length $k$ ) is a sequence $x_{0}, x_{1}, \ldots, x_{k}$, where $x_{j} \rightarrow x_{j+1}, 0 \leq j<k$. A walk (of length $k$ ) is a sequence $x_{0}, x_{1}, \ldots, x_{k}$, where $x_{j} \rightarrow x_{j+1}$ or $x_{j+1} \rightarrow x_{j}, 0 \leq j<k$. A connected (strongly connected, respectively) component of a graph is a maximal subset in which every pair of nodes is connected by a walk (path, respectively). Components form a partition of the nodes of a graph. A graph is (strongly) connected if there is a single (strongly) connected component, that is, for every choice of $x$ and $y$ there is a walk (path) from $x$ to $y$. A strongly connected component is terminal if its nodes have no arc towards other components.

The distance $d(x, y)$ from $x$ to $y$ is the length of a shortest path from $x$ to $y$, or $\infty$ if no such path exists. The nodes reachable from $x$ are the nodes $y$ such that $d(x, y)<\infty$. The nodes coreachable from $x$ are the nodes $y$ such that $d(y, x)<\infty$. A node has trivial (co)reachable set if the latter contains only the node itself.

Let $A$ be a nonnegative matrix. We will denote with $\rho(A)$ the spectral radius of $A$, that is, the modulo of the largest eigenvalue. By Perron-Frobenius theory (Berman \& Plemmons, 1994), $\rho(A)$ is an eigenvalue of $A$. It is called the dominant eigenvalue, and the associated eigenvectors are called dominant eigenvectors. The notation $\bar{A}$ will be used throughout the paper to denote the matrix obtained by $\ell_{1}$-normalizing the rows of $A$, that is, dividing each element of a row by the sum of the row (null rows are left unchanged). If there are no null

4 Sabidussi's paper tried to capture rank monotonicity, too, but with much weaker requirements: he only required that if $y$ has maximum score in the network, then it should have maximum score also after the addition of an arc towards $y$. 
rows, $\bar{A}$ is (row-)stochastic, that is, it is nonnegative and all rows sum to one; in this case, its spectral radius is 1 . Unless otherwise specified, all vectors in this paper are row vectors.

\subsection{Centrality measures}

In this section we quickly sketch the mathematical definitions of the centrality measures studied in this paper. The reader can find an accurate historical account in (Boldi \& Vigna, 2014), along with motivations. When several alternative definitions are possible we report the one that is better suited to our proofs.

Geometric measures assume that importance is a function of distances. In particular, we have:

Indegree. The number of incoming arcs $d^{-}(x)$, that is, the nodes whose distance to $x$ is one.

Closeness. Bavelas (1948) introduced closeness in the late forties; the closeness of $x$ is defined by

$$
\frac{1}{\sum_{y} d(y, x)} .
$$

For this definition to make sense, the graph must be strongly connected. Lacking that condition, some of the summands will be $\infty$, resulting in a null score for all nodes that cannot coreach the whole graph. Often closeness is patched by eliminating infinite summands at the denominator, and this is the definition we shall use in the rest of the paper.

Lin's index. Nan Lin (1976) tried to repair the definition of closeness for graphs with infinite distances by weighting closeness using the square of the number of coreachable nodes; his definition for the centrality of a node $x$ with a nonempty coreachable set is

$$
\frac{|\{y \mid d(y, x)<\infty\}|^{2}}{\sum_{d(y, x)<\infty} d(y, x)} .
$$

Harmonic centrality. Closeness is essentially the reciprocal of a denormalized arithmetic mean. If we instead take the reciprocal of a denormalized harmonic mean we obtain harmonic centrality:

$$
\sum_{y \neq x} \frac{1}{d(y, x)} .
$$

Spectral measures compute the dominant left eigenvector of some matrix derived from the adjacency matrix $A$ of the graph, and depending on how the matrix is modified before the computation we can obtain a number of different measures. In general, such vectors are defined up to a positive multiplicative constant, and are unique if the matrix is irreducible (Berman \& Plemmons, 1994), which is true of the adjacency matrix $A$ iff the graph is strongly connected.

The dominant left eigenvector. The dominant left eigenvector of the adjacency matrix A (Wei, 1952; Berge, 1958).

Seeley's index. The dominant left eigenvector of $\bar{A}$, the adjacency matrix with $\ell_{1}$-normalized rows (Seeley, 1949). It is the stationary state of the natural Markov chain on the graph, in which the next state is chosen uniformly among successors. 
Katz's index. A recursive summation of paths, that can be seen as a linear operator:

$$
\mathbf{1} \sum_{i=0}^{\infty} \beta^{i} A^{i}=\mathbf{1}(1-\beta A)^{-1},
$$

where $\beta$ is a parameter satisfying the condition $0 \leq \beta<1 / \rho(A)$ (Katz, 1953). The constant vector 1 can be replaced by a positive preference vector (Hubbell, 1965).

PageRank. A recursive sum of weighted paths (Page et al., 1998), that again can be seen as a linear operator:

$$
\boldsymbol{p}=(1-\alpha) \boldsymbol{v} \sum_{i=0}^{\infty} \alpha^{i} \bar{A}^{i}=(1-\alpha) \boldsymbol{v}(1-\alpha \bar{A})^{-1} .
$$

Here $\boldsymbol{v}$ is a preference vector (a probability distribution) and $0 \leq \alpha<1$.

HITS. The dominant right singular vector of $A$, which is just the dominant left eigenvector of $A^{T} A$ (Bonacich, 1991; Kleinberg, 1999).

SALSA. The dominant left eigenvector of $\overline{A^{T}} \bar{A}$ (Lempel \& Moran, 2001).

Note that in the last two cases one can also consider the alternative score given by the left dominant vector of $A A^{T}$ (indeed, usually the two scores are used at the same time). The two eigenvectors are exchanged when the graph is transposed, so we need to discuss just one.

Finally, betweenness centrality was introduced by Anthonisse (1971) under the name of rush and rediscovered later (Freeman, 1977). The idea is to measure the probability that a random shortest path passes through a given node: if $\sigma_{y z}$ is the number of shortest paths going from $y$ to $z$, and $\sigma_{y z}(x)$ is the number of such paths that pass through $x$, we define the betweenness of $x$ as

$$
\sum_{y, z \neq x, \sigma_{y z} \neq 0} \frac{\sigma_{y z}(x)}{\sigma_{y z}} .
$$

\section{Rank Monotonicity}

We assume from the beginning that the centrality measures under examination are invariant by isomorphism, that is, that they depend just on the structure of the graph, and not on particular labeling chosen for each node: all measures defined above are such (this condition is sometimes called anonymity).

Boldi and Vigna (2014) proposed to study the following property:

Definition 1 (Score-Monotonicity Axiom) A centrality measure satisfies the score-monotonicity axiom if for every graph $G$ and every pair of nodes $x, y$ such that $x \neq y$, when we add $x \rightarrow y$ to $G$ the centrality of $y$ increases.

Score monotonicity is just half of what one really wants: it is indeed possible to define pathological scores that are score monotone, but modify the relative score of nodes in counterintuitive ways. For example, you may find scenarios where the score of a node $z$, originally smaller than that of $y$, is made larger than that of $y$ by the addition of the arc $x \rightarrow y$. To avoid these problems, we suggest to study the following natural axiom, which appears in the work of Chien, Dwork, Kumar, Simon and Sivakumar (2004): 
Definition 2 (Rank-Monotonicity Axiom) A centrality measure satisfies the rank-monotonicity axiom if for every graph $G$ and every pair of nodes $x, y$ such that $x \rightarrow y$, when we add $x \rightarrow y$ to $G$ the following happens:

- if the score of $z$ was strictly smaller than the score of $y$, this fact remains true after adding $x \rightarrow y$;

- if the score of $z$ was smaller than or equal to the score of $y$, this remains true after adding $x \rightarrow y$.

Note that another formulation of the above definition is as follows:

- if the score of $z$ was strictly smaller than the score of $y$, this remains true after adding $x \rightarrow y$;

- if the score of $z$ was equal to the score of $y$, it remains equal o becomes smaller after adding $x \rightarrow y$.

We also define the strict version:

Definition 3 (Strict Rank-Monotonicity Axiom) A centrality measure satisfies the strict rank-monotonicity axiom if for every graph $G$ and every pair of nodes $x, y$ such that $x \neq t y$, when we add $x \rightarrow y$ to $G$ the following happens:

- if the score of $z \neq y$ was smaller than or equal to the score of $y$, after adding $x \rightarrow y$ the score of $z$ becomes smaller than the score of $y$.

Note that the only difference between the last two definitions is the behavior on ties (nodes with the same score as $y$ ): if a measure is strictly rank monotone, adding an $\operatorname{arc} x \rightarrow y$ will break all ties with other nodes in favor of $y$.

\section{Proofs and Counterexamples}

We have now defined a number of centrality measures and two axioms. We are thus going to provide a proof or a counterexample for each combination. We will also pose each question in two different environments: general graphs, and strongly connected graphs. To make the treatment complete, we will try to provide answers for the score-monotonicity axiom on strongly connected graphs when necessary (i.e., when the axiom is not satisfied in general graphs).

\subsection{Harmonic centrality}

It is easy to see that harmonic centrality satisfies score monotonicity on all graphs: as we will see, it is also strictly rank monotone. We start by proving a lemma which bounds the variation of additive contributions in the definition of harmonic centrality:

Lemma 1 Let $G$ be a graph with distance function $d$, and let $d^{\prime}$ be the distance function of $G$ with an additional new arc $x \rightarrow y, x \neq y$. Then, for every node $w \neq y$ and $z \neq w$ we have

$$
\frac{1}{d^{\prime}(w, z)}-\frac{1}{d(w, z)} \leq \frac{1}{d^{\prime}(w, y)}-\frac{1}{d(w, y)} .
$$




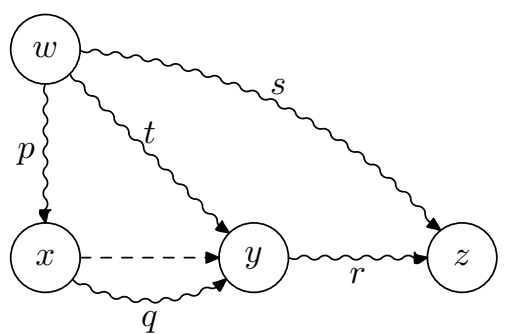

Fig. 1. Typical node configuration for geometric measures. The path labels represent the length of a shortest path.

Moreover, if $d^{\prime}(w, z)<d(w, z)$

$$
\frac{1}{d^{\prime}(w, z)}-\frac{1}{d(w, z)}<\frac{1}{d^{\prime}(w, y)}-\frac{1}{d(w, y)} .
$$

In particular for all nodes $z \neq x, y$,

$$
\frac{1}{d^{\prime}(x, z)}-\frac{1}{d(x, z)}<\frac{1}{d^{\prime}(x, y)}-\frac{1}{d(x, y)}
$$

Proof

The first part of the thesis is obvious if $d^{\prime}(w, z)=d(w, z)$. Otherwise, with the notation of Figure 1, the hypothesis $d^{\prime}(w, z)<d(w, z)$ yields $s>p+1+r$ (which implies $p, r<$ $\infty)$. Note that in this case $t>p+1$, as otherwise $s>p+1+r \geq t+r$, contradicting the triangular inequality $s \leq t+r$. We conclude that

$$
\frac{1}{d^{\prime}(w, z)}-\frac{1}{d(w, z)}=\frac{1}{p+1+r}-\frac{1}{s}<\frac{1}{p+1}-\frac{1}{t}=\frac{1}{d^{\prime}(w, y)}-\frac{1}{d(w, y)},
$$

since when $s, t<\infty$

$$
\frac{1}{p+1+r}-\frac{1}{s}-\left(\frac{1}{p+1}-\frac{1}{t}\right)=\frac{p+1-p-1-r}{(p+1+r)(p+1)}+\frac{s-t}{s t}<-\frac{r}{s t}+\frac{r}{s t}=0,
$$

and the remaining cases (where $s$ or $t$ are infinite) are trivial.

Theorem 1 Harmonic centrality satisfies strict rank monotonicity on all graphs.

Proof

With the notation of Lemma 1, we assume that for a node $z \neq y$

$$
\sum_{w \neq z} \frac{1}{d(w, z)} \leq \sum_{w \neq y} \frac{1}{d(w, y)} .
$$

Adding the latter inequality to that of Lemma 1 , for every $w \neq y, z$, we obtain

$$
\sum_{w \neq z, y} \frac{1}{d^{\prime}(w, z)}+\frac{1}{d(y, z)} \leq \sum_{w \neq z, y} \frac{1}{d^{\prime}(w, y)}+\frac{1}{d(z, y)}
$$

Now, obviously $d^{\prime}(y, z)=d(y, z)$ and $d^{\prime}(z, y) \leq d(z, y)$. But then either $z \neq x$, in which case at least for $w=x$ we are adding a strict inequality (the last inequality in the statement of Lemma 1), or $z=x$, in which case $d^{\prime}(z, y)<d(z, y)$. This concludes the proof. 


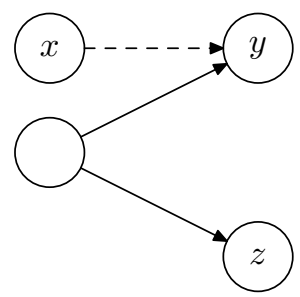

Fig. 2. A counterexample showing that closeness does not satisfy rank monotonicity on general graphs.

\subsection{Closeness}

Closeness does not satisfy score monotonicity in general (Boldi \& Vigna, 2014), but it is trivial to show that it does on strongly connected graphs. It is also easy to show that it does not satisfy rank monotonicity in general graphs: a simple counterexample is shown in Figure 2. ${ }^{5}$ Both node $y$ and node $z$ have closeness equal to 1 , as the summation in (1) reduces to a single summand of value 1 . When we add an arc from $x$ to $y$, the score of $z$ does not change, but the score of $y$ becomes $1 / 2$, as now we are adding two summands of value 1 .

Nonetheless, things improve on strongly connected graphs, where we can prove a result analogous to (but weaker than) Lemma 1:

Lemma 2 Let $G$ be a graph with distance function $d$, and let $d^{\prime}$ be the distance function of $G$ with an additional new arc $x \rightarrow y$. Then, for every nodes $w$ and $z$

$$
d(w, z)-d^{\prime}(w, z) \leq d(w, y)-d^{\prime}(w, y) .
$$

Proof

If $d(w, z)=d^{\prime}(w, z)$ the thesis is obvious. Otherwise, looking at Figure 1, we have $s \leq t+r$ by the triangular inequality. Thus,

$$
d(w, z)-d^{\prime}(w, z) \leq s-p-1-r \leq t-p-1 \leq d(w, y)-d^{\prime}(w, y) .
$$

At this point, a proof analogous to that of Theorem 1 shows that

Theorem 2 Closeness satisfies rank monotonicity on strongly connected graphs.

Proof

With the notation of Lemma 2, we assume that for a node $z$

$$
\frac{1}{\sum_{w} d(w, z)} \leq \frac{1}{\sum_{w} d(w, y)} .
$$

Equivalently,

$$
\sum_{w} d(w, y) \leq \sum_{w} d(w, z)
$$

5 We remark that it is easy to show that score and rank monotonicity are not satisfied on general graphs even if infinite distances are not eliminated from the summation. 


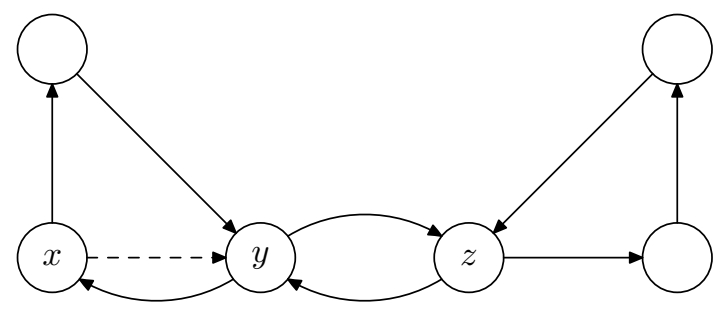

Fig. 3. A counterexample showing that closeness does not satisfy strict rank monotonicity on strongly connected graphs.

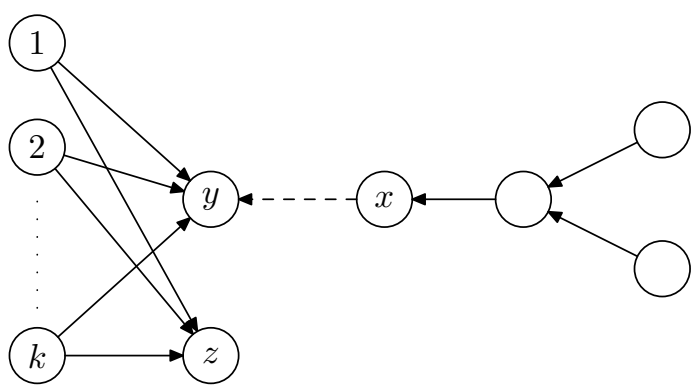

Fig. 4. A counterexamples showing that Lin's index does not satisfy rank monotonicity on general graphs.

and adding for all $w$ the inequalities of Lemma 2

$$
\sum_{w} d^{\prime}(w, y) \leq \sum_{w} d^{\prime}(w, z)
$$

The same chain of deductions is true if we start from a strict inequality. A final inversion completes the proof.

The previous theorem cannot be improved to strict rank monotonicity, as the example in Figure 3 shows. The rank of $y$ and $z$ in the graph is the same (by symmetry) before adding the arc $x \rightarrow y$, but it remains the same after the addition. Indeed, for what concerns $y$ the only effect on the summation in (1) of the new arc is that of changing the distance from $x$ to $y$ from 2 to 1 , thus reducing the summation by 1 . On the other hand, for what concerns $z$ the only effect of the new arc is that of changing the distance from $x$ to $z$ from 3 to 2, once again reducing the summation by 1 .

\subsection{Lin's index}

Lin's index does not satisfy score monotonicity in general (Boldi \& Vigna, 2014), but on strongly connected graph it is equivalent to closeness, so it satisfies score and rank monotonicity (but not strict rank monotonicity). It is again easy to show that it does not satisfy rank monotonicity in general graphs: a counterexample is shown in Figure 4. The Lin centrality of $y$ and $z$ is $(k+1)^{2} / k$. After adding an arc $x \rightarrow y$, the centrality of $y$ becomes $(k+5)^{2} /(k+9)$, which is smaller than the previous value when $k>3$, and thus smaller than the centrality score of $z$, which does not change. 


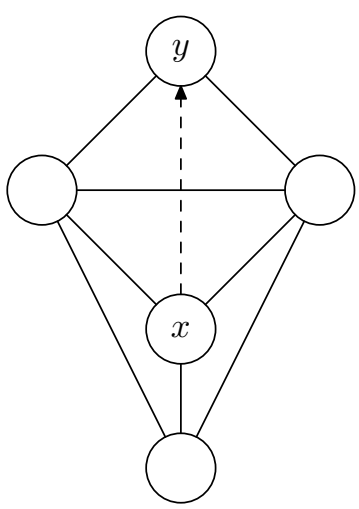

Fig. 5. A counterexample showing that betweenness satisfies neither score nor rank monotonicity on strongly connected graphs.

\subsection{Betweenness}

Betweenness centrality fails to satisfy all axioms of (Boldi \& Vigna, 2014). It also fails to satisfy rank monotonicity, even on strongly connected graphs. In Figure 5 we show a graph $G$ (for simplicity, we represent by an edge a pair of symmetric arcs) to which an arc $x \rightarrow y$ is added, getting the graph $G^{\prime}$.

In $G$, the score of $x$ and $y$ is zero, since there is no shortest path passing through them. But when we add the arc $x \rightarrow y$, a new shortest path arises through $x$, raising its score to $1 / 3$, but not through $y$, so its score remains zero. As a result, rank monotonicity and score monotonicity are both violated on a strongly connected graph.

\subsection{PageRank and Katz's index}

Rank monotonicity of PageRank was proved by Chien, Dwork, Kumar, Simon and Sivakumar (2004). Their proof works for a generic regular Markov chain: in the case of PageRank this condition is true, for instance, if the preference vector is strictly positive or if the graph is strongly connected.

\subsubsection{Properties of damped spectral rankings}

In this work, we aim at lifting almost all hypothesis on the underlying graph and preference vector, and at showing a stronger result: strict rank monotonicity. In fact, we prove (strict) rank monotonicity for certain updates of a generic damped spectral ranking given by

$$
\boldsymbol{r}=\boldsymbol{v} \sum_{n \geq 0}(\alpha M)^{n}=\boldsymbol{v}(1-\alpha M)^{-1}
$$

where $M$ is a nonnegative matrix, $0 \leq \alpha<1 / \rho(M)$ is a damping factor, and $v$ is a nonnegative preference vector. Both PageRank and Katz's index are special instances of damped spectral ranking.

In order to prove our result, we first need the following: 
Lemma 3 Let $M$ be a nonnegative matrix, $0 \leq \alpha<1 / \rho(M)$ a damping factor and $v a$ nonnegative preference vector. Let

$$
\boldsymbol{r}=\boldsymbol{v} \sum_{n \geq 0}(\alpha M)^{n}
$$

be the associated damped spectral ranking and let $C=(1-\alpha M)^{-1}$. Then, given $y$ and $z$ such that $c_{y z}>0$ and letting $q=c_{y y} / c_{y z}$, we have $c_{w y} \leq q \cdot c_{w z}$ for all $w$. In particular, if $r_{y} \neq 0$

- if $r_{z} \leq r_{y}$, then $c_{y z} \leq c_{y y}$;

- if $r_{z}<r_{y}$, then $c_{y z}<c_{y y}$.

Proof

The first claim is a restatement of the known property (Willoughby, 1977) that for all $y, z$ and $w$

so

$$
c_{w z} \geq \frac{c_{w y} c_{y z}}{c_{y y}},
$$

$$
q c_{w z} \geq c_{w y} .
$$

Note now that if $c_{y y}<c_{y z}$, then $q<1$, and

$$
r_{y}=\sum_{w} v_{w} c_{w y}<\sum_{w} v_{w} c_{w z}=r_{z},
$$

which proves the first item (the strict inequality is due to the assumption $r_{y} \neq 0$ ). If $c_{y y} \leq c_{y z}$, then $q \leq 1$, and the second item follows similarly.

Note that the hypothesis on $r_{y}$ is necessary: consider the matrix

$$
M=\left(\begin{array}{lll}
1 & 1 & 0 \\
1 & 0 & 0 \\
0 & 0 & 1
\end{array}\right),
$$

whose spectral radius is the golden ratio $(1+\sqrt{5}) / 2$; for $\alpha=3 / 5$ we have

$$
\sum_{n \geq 0}(\alpha M)^{n}=\left(\begin{array}{rrr}
25 & 15 & 0 \\
15 & 10 & 0 \\
0 & 0 & \frac{5}{2}
\end{array}\right) .
$$

If we consider the preference vector $\langle 0,0,1\rangle$, the associated spectral ranking will be $\langle 0,0,5 / 2\rangle$. If we let $z$ and $y$ be the first and second node, respectively, we have $c_{y z}=15>0,0=r_{y} \leq$ $r_{z}=0$ but $c_{y z}=15>10=c_{y y}$, showing that Lemma 3 would not be true if the requirement $r_{y} \neq 0$ was dropped.

We can finally prove our main theorem:

Theorem 3 Let $M$ and $M^{\prime}$ be two nonnegative matrices, such that $M^{\prime}-M=\boldsymbol{\chi}_{x}^{T} \boldsymbol{\delta}$ (i.e., the matrices differ only on the $x$-th row, and $\boldsymbol{\delta}$ is the corresponding row difference). Let also $\boldsymbol{v}$ be a nonnegative preference vector and $0 \leq \alpha<\min \left(1 / \rho(M), 1 / \rho\left(M^{\prime}\right)\right)$; let $\boldsymbol{r}$ and $\boldsymbol{r}^{\prime}$ be the damped spectral rankings (4) associated with $M$ and $M^{\prime}$ respectively. Assume further that: 
1. there is exactly one $y$ such that $\delta_{y}>0$;

2. $r_{y} \neq 0$

3. $r_{y} \leq r_{y}^{\prime}$.

Then, if $r_{z} \leq r_{y}$ we have $r_{z}^{\prime}-r_{z} \leq r_{y}^{\prime}-r_{y}$. As a consequence, $r_{z} \leq r_{y}$ implies $r_{z}^{\prime} \leq r_{y}^{\prime}$, whereas $r_{z}<r_{y}$ implies $r_{z}^{\prime}<r_{y}^{\prime}$.

Proof

In this proof, as in the Lemma, we let $C=(1-\alpha M)^{-1}$. First of all, we note that given the hypotheses both $1-\alpha M$ and $1-\alpha M^{\prime}$ are $M$-matrices, so they both have positive determinants. Since $M^{\prime}$ is obtained from $M$ by a rank-one correction $\left(M^{\prime}=M+\boldsymbol{\chi}_{x}^{T} \boldsymbol{\delta}\right)$, applying the matrix determinant lemma we have

$$
\operatorname{det}\left(1-\alpha M^{\prime}\right)=\operatorname{det}\left(1-\alpha M-\alpha \boldsymbol{\chi}_{x}^{T} \boldsymbol{\delta}\right)=\left(1-\alpha \boldsymbol{\delta}(1-\alpha M)^{-1} \boldsymbol{\chi}_{x}^{T}\right) \operatorname{det}(1-\alpha M) .
$$

We conclude that necessarily

$$
1-\alpha \boldsymbol{\delta}(1-\alpha M)^{-1} \boldsymbol{\chi}_{x}^{T}>0 .
$$

We now use the Sherman-Morrison formula to write down the inverse of $1-\alpha M^{\prime}$ as a function of $1-\alpha M$. More precisely,

$$
\begin{aligned}
\left(1-\alpha M^{\prime}\right)^{-1}=\left(1-\alpha\left(M+\boldsymbol{\chi}_{x}^{T} \boldsymbol{\delta}\right)\right)^{-1} & =\left(1-\alpha M-\alpha \boldsymbol{\chi}_{x}^{T} \boldsymbol{\delta}\right)^{-1} \\
= & (1-\alpha M)^{-1}+\frac{(1-\alpha M)^{-1} \alpha \boldsymbol{\chi}_{x}^{T} \boldsymbol{\delta}(1-\alpha M)^{-1}}{1-\alpha \boldsymbol{\delta}(1-\alpha M)^{-1} \boldsymbol{\chi}_{x}^{T}} .
\end{aligned}
$$

We now multiply by the preference vector $v$, obtaining the explicit spectral-rank correction:

$$
\begin{gathered}
\boldsymbol{r}^{\prime}=\boldsymbol{v}\left(1-\alpha M^{\prime}\right)^{-1}=\boldsymbol{v}(1-\alpha M)^{-1}+\boldsymbol{v} \frac{(1-\alpha M)^{-1} \alpha \boldsymbol{\chi}_{x}^{T} \boldsymbol{\delta}(1-\alpha M)^{-1}}{1-\alpha \boldsymbol{\delta}(1-\alpha M)^{-1} \boldsymbol{\chi}_{x}^{T}} \\
=\boldsymbol{r}+\frac{\alpha \boldsymbol{r} \boldsymbol{\chi}_{x}^{T} \boldsymbol{\delta}(1-\alpha M)^{-1}}{1-\alpha \boldsymbol{\delta}(1-\alpha M)^{-1} \boldsymbol{\chi}_{x}^{T}}=\boldsymbol{r}+\frac{\alpha r_{x}}{1-\alpha \boldsymbol{\delta}(1-\alpha M)^{-1} \boldsymbol{\chi}_{x}^{T}} \boldsymbol{\delta}(1-\alpha M)^{-1} .
\end{gathered}
$$

The case $r_{x}=0$ is obvious. Thus, let us assume that $r_{x}>0$. By (6), we can gather all the scalar values appearing in the second summand into a single positive constant $\kappa$ and just write

$$
\boldsymbol{r}^{\prime}-\boldsymbol{r}=\kappa \boldsymbol{\delta}(1-\alpha M)^{-1}
$$

Note that if

$$
\left[\boldsymbol{\delta}(1-\alpha M)^{-1}\right]_{z} \leq 0
$$

the thesis is trivial by the hypothesis $r_{y} \leq r_{y}^{\prime}$. This holds true, in particular, if $c_{y z}=0$, as in that case

$$
\left[\boldsymbol{\delta}(1-\alpha M)^{-1}\right]_{z}=-\sum_{w \neq y}\left|\delta_{w}\right| c_{w z} \leq 0
$$




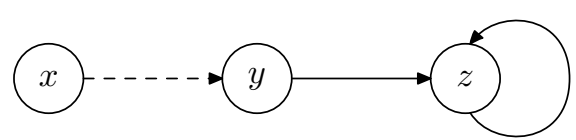

Fig. 6. A counterexample for the last hypotheses of Theorem 3.

If $c_{y z}>0$, since $r_{y} \neq 0$ we know from Lemma 3 that $q=c_{y y} / c_{y z} \geq 1$, and for all $w$ we have $c_{w y} \leq q c_{w z}$. It follows that

$$
\begin{aligned}
{\left[\boldsymbol{\delta}(1-\alpha M)^{-1}\right]_{y} } & =\delta_{y} c_{y y}-\sum_{w \neq y}\left|\delta_{w}\right| c_{w y} \geq \delta_{y} q c_{y z}-\sum_{w \neq y} q\left|\delta_{w}\right| c_{w z} \\
& =q\left(\delta_{y} c_{y z}-\sum_{w \neq y}\left|\delta_{w}\right| c_{w z}\right)=q\left[\boldsymbol{\delta}(1-\alpha M)^{-1}\right]_{z} \geq\left[\boldsymbol{\delta}(1-\alpha M)^{-1}\right]_{z}
\end{aligned}
$$

This completes the proof.

We remark that no hypothesis in the statement of the last theorem can be weakened. The update vector must increase a single coordinate to model an increase of importance of $y$ alone.

The condition $r_{y} \neq 0$ cannot be weakened, as the counterexample shown in Figure 6 proves. The spectral ranking $\boldsymbol{r}$ induced by the adjacency matrix with preference vector $\langle 1,0,0\rangle$, without the dotted arrow, has $r_{y}=r_{z}=0$. If we add the dotted arrow, though, the score vector becomes $\boldsymbol{r}^{\prime}=\left\langle 1, \alpha, \alpha^{2} /(1-\alpha)\right\rangle$, and $r_{z}^{\prime}-r_{z}=r_{z}^{\prime}=\alpha^{2} /(1-\alpha)$ is larger than $r_{y}^{\prime}-r_{y}=r_{y}^{\prime}=\alpha$ for $\alpha>1 / 2$.

Finally, the condition $r_{y} \leq r_{y}^{\prime}$ cannot be eliminated. Consider once again the matrix $M$ of (5), and its spectral rank with $\alpha=3 / 5$ and preference vector $\langle 1,1,4\rangle$, which is $\langle 40,25,10\rangle$. If we update the second row using the vector $\langle-1,1,0\rangle$ the new scores will be $\langle 5 / 2,25 / 4,10\rangle$ contradict the thesis.

Note, however, that we can actually prove the condition under mild assumptions on $M$ and $\boldsymbol{\delta}$ :

Theorem 4 Condition (3) of Theorem 3 can be substituted by the following two hypotheses (that imply it)

1. $1-\alpha M$ is (strictly) diagonally dominant

2. $\sum_{z} \delta_{z} \geq 0$.

Moreover, in the strict case, $r_{y}<r_{y}^{\prime}$, provided that $r_{x}>0$.

Proof

The proof follows the lines of the proof of Theorem 3, noting again that for $r_{x}=0$ the statement trivializes. However, once we get the update vector $\boldsymbol{\delta}$ we now note that being $1-\alpha M$ diagonally dominant, the (nonnegative) inverse $C=(1-\alpha M)^{-1}$ has the property that the entries $c_{i i}$ on the diagonal are (strictly) larger than off-diagonal entries $C_{k i}$ on the same column, as shown in Remark 3.3 of (McDonald et al., 1995). Thus,

$$
\left[\boldsymbol{\delta}(1-\alpha M)^{-1}\right]_{y}=\delta_{y} c_{y y}-\sum_{z \neq y}\left|\delta_{z}\right| c_{z y} \geq \delta_{y} c_{y y}-\sum_{z \neq y}\left|\delta_{z}\right| c_{y y} \geq 0 .
$$


In the strict case, if there is at least one index $z \neq y$ such that $\left|\delta_{z}\right| c_{z y} \neq 0$, then the first inequality is strict; otherwise, the second inequality is strict (because $\delta_{y}>0$ and $c_{y y}>0$ ).

Finally, we can prove strict rank monotonicity under the additional hypothesis of score monotonicity:

Theorem 5 Let $M$ and $M^{\prime}$ be two nonnegative matrices as in Theorem 3 and let $\boldsymbol{r}$ and $\boldsymbol{r}^{\prime}$ be the damped spectral rankings associated to $M$ and $M^{\prime}$ respectively. Assume further that:

1. there is exactly one $y$ such that $\delta_{y}>0$;

2. $r_{x}, r_{y} \neq 0$

3. $r_{y}<r_{y}^{\prime}$.

Then, if $r_{z} \leq r_{y}$ we have $r_{z}^{\prime}-r_{z}<r_{y}^{\prime}-r_{y}$, and in particular $r_{z}^{\prime}<r_{y}^{\prime}$.

The proof is the same as that of Theorem 3: the additional hypotheses makes it possible to make the relevant inequalities strict.

\subsubsection{Applications to PageRank and Katz's index}

These results on damped spectral rankings show, in particular, that all known variants of PageRank (strongly preferential, weakly preferential, pseudoranks, etc.) and Katz's index cannot be proved to satisfy rank monotonicity for an arbitrary preference vector without additional hypotheses (e.g., that all scores are positive).

Corollary 1 PageRank satisfies the strict rank-monotonicity axiom, for any graph, damping factor and preference vector, provided all scores are nonzero. The latter condition is always true if the preference vector is everywhere nonzero or if the graph is strongly connected.

Proof

Consider two nodes $x$ and $y$ of a graph $G$ such that there is no arc from $x$ to $y$, and let $d$ be the outdegree of $x$. Given the normalized matrix $\bar{A}$ of $G$, and the normalized matrix $\bar{A}^{\prime}$ of the graph $G^{\prime}$ obtained by adding to $G$ the $\operatorname{arc} x \rightarrow y$, we have

$$
\bar{A}^{\prime}-\bar{A}=\boldsymbol{\chi}_{x}^{T} \boldsymbol{\delta},
$$

where $\boldsymbol{\delta}$ is the difference between the rows corresponding to $x$ in $\bar{A}$ and $\bar{A}^{\prime}$, which contains $-1 / d(d+1)$ in the positions corresponding to the successors of $x$ in $G$, and $1 /(d+1)$ in the position corresponding to $y$ (note that if $d=0$, we have just the latter entry), so we can apply Theorem 5 . The hypothesis $r_{y}<r_{y}^{\prime}$ is always verified by Theorem 4 .

Corollary 2 Katz's index satisfies the strict rank-monotonicity axiom, for any graph, attenuation factor and preference vector, provided all scores are nonzero. The latter condition is always true if the preference vector is everywhere nonzero or if the graph is strongly connected.

Proof 
Consider two nodes $x$ and $y$ of a graph $G$ such that there is no arc from $x$ to $y$. Given the matrix $A$ of $G$, and the matrix $A^{\prime}$ of the graph $G^{\prime}$ obtained by adding to $G$ the $\operatorname{arc} x \rightarrow y$, we have

$$
A^{\prime}-A=\boldsymbol{\chi}_{x}^{T} \boldsymbol{\chi}_{y}
$$

and we can apply Theorem 3. The hypothesis $r_{y}<r_{y}^{\prime}$ is trivially verified, as the only nonzero entry of $\boldsymbol{\delta}=\boldsymbol{\chi}_{y}$ is the positive one.

It is interesting to note there is a kind of duality between the hardness in proving score monotonicity in (Boldi \& Vigna, 2014) and strict rank monotonicity here. When proving score monotonicity, the problem is that $r_{x}$ must be nonzero, or the score of $y$ will not increase. When proving rank monotonicity, instead, $r_{x}=0$ is not an issue: but if $r_{y}=0$, we have no way to control the growth in score of other nodes with respect to $y$, as (5) shows.

\section{Other dominant eigenvectors}

The definition of Katz's index and PageRank is free of multiplicative constants. This is no longer true of the other spectral measures based on dominant eigenvectors introduced in Section 3. Thus, proving or disproving statements about these measures requires some care.

There is a common theme to all counterexamples: a component is built into the example in such a way that it absorbs all the rank of the dominant left eigenvector (i.e., all other nodes have a zero score). This can be crafted by making the component sufficiently dense (i.e., a small clique) and usually terminal in the graph of strongly connected components. Another node $y$ is placed in such a way that connecting the component to the node makes the graph strongly connected. However, we make the graph somewhat dense around another node $z$, which causes the score of $z$ to become larger than that of $y$ when this happens.

\subsection{Seeley's index and SALSA}

For Seeley's index and SALSA, we can avoid multiplicative constants. In the first case, we will restrict our considerations to graphs in which all nodes have at least an outgoing link, and there is exactly one terminal strongly connected component. The associated matrices are stochastic and the resulting Markov chains have a unique stationary state, which makes it possible to define a unique, $\ell_{1}$-normalized dominant eigenvector (i.e., make it into a probability distribution). The restrictions are sufficient to prove or disprove all our results.

In the case of SALSA (Lempel \& Moran, 2001), there is a closed form expression for the score: one computes the connected components of the symmetric graph induced by the matrix $A^{T} A$; the SALSA score of a node is the ratio between its indegree and the sum of the indegrees of nodes in the same component of the symmetric graph, multiplied by the ratio between the component size and the graph size. Also in this case, the resulting vector is $\ell_{1}$-normalized.

In both cases, the vector we define is equivalent to the limit obtained starting from the uniform distribution and iterating multiplication by the matrix defining the score (in the case of SALSA, assuming that every node has at least an incoming and an outgoing link), which makes the choice natural. 


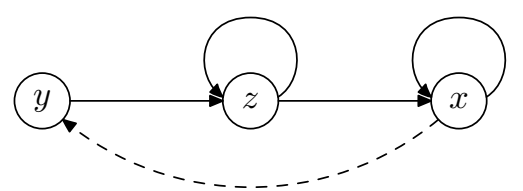

Fig. 7. A counterexample for rank monotonicity of Seeley's index on general graphs.

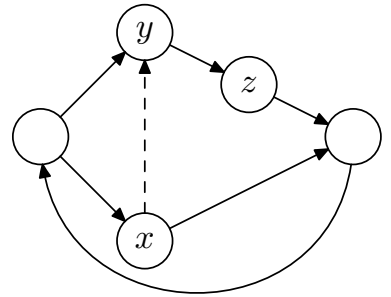

Fig. 8. A counterexample for strict rank monotonicity of Seeley's index on strongly connected graphs.

Using the definition above, we can show that Seeley's index is not rank monotone on general graphs. Consider the counterexample in Figure 7: before adding the arc $x \rightarrow y$, there is a unique dominant left eigenvector that is zero on $y$ and $z$. After adding the arc, the dominant left eigenvector is $\langle 1 / 5,2 / 5,2 / 5\rangle$. Incidentally, the counterexample shows also that Seeley's index is not score monotone, as adding an arc from $z$ to $y$ will leave the dominant left eigenvector unchanged.

On the other hand, on strongly connected graphs Seeley's index is the stationary state of a regular Markov chain, and we can apply the results of (Chien et al., 2004), which prove score and rank monotonicity. Nonetheless, Seeley's index does not satisfy strict rank monotonicity: in Figure 8 we show a graph in which the score of $y$ and $z$ is equal. By the dominant left eigenvector equation (i.e., the stationary state equation), this happens whenever a node of outdegree one has an arc towards a node of indegree one. So when we add an arc $x \rightarrow y$, the score of $y$ increases, but the score of $z$ remains equal to the score of $y$.

For what matters SALSA, Figure 9 shows a counterexample that violates both score and rank monotonicity on a strongly connected graph: before adding the arc $x \rightarrow y$, all scores are equal to $1 / 8$; after adding $x \rightarrow y$, the score of $y$ decreases to $3 / 28$ and the score of $z$ increases to $3 / 14$.

\subsection{The dominant left eigenvector and HITS}

In the first part of this section, we will describe a number of negative results. We will define graphs such that the associated scores will be always unique, which will relieve us from the problem of multiple dominant eigenvectors. We will also circumvent the problem of multiplicative constants by using zero-vs-nonzero arguments.

First of all, the counterexample in Figure 10 shows that the dominant left eigenvector is not rank monotone on general graphs. Before adding the arc $x \rightarrow y$, there is a unique dominant left eigenvector that is zero on $y$ and $z$ and has the same value on all other nodes. After adding the arc, the dominant left eigenvector gives to $z$ a centrality greater than $y$. 


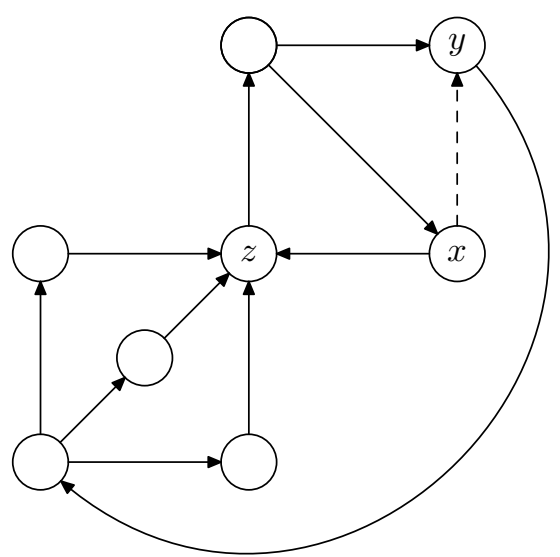

Fig. 9. A counterexample for score and rank monotonicity of SALSA on strongly connected graphs.

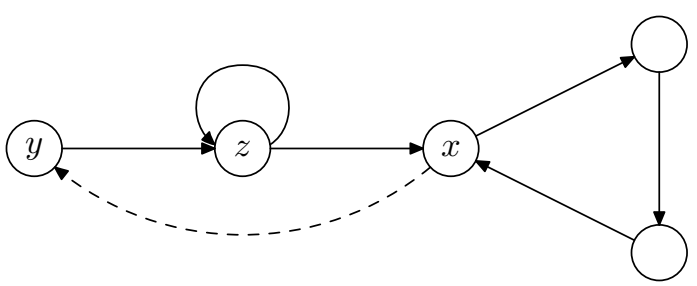

Fig. 10. A counterexample for rank monotonicity of the dominant left eigenvector on general graphs.

The counterexample in Figure 11 shows that HITS is not rank monotone on strongly connected graphs. Before adding the arc $x \rightarrow y$, there is a unique dominant left eigenvector that is zero on all nodes except those of the 3 -clique. After adding the arc, HITS gives to $z$ a centrality greater than $y$.

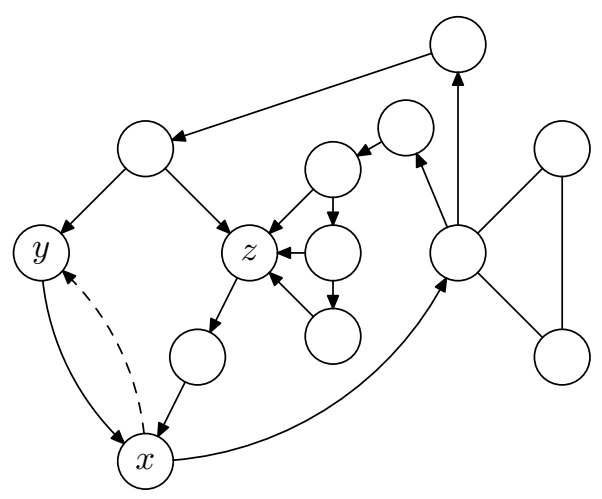

Fig. 11. A counterexample for rank monotonicity of HITS on strongly connected graphs. 


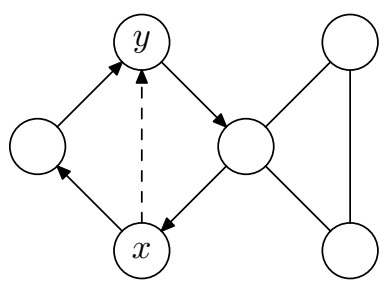

Fig. 12. A counterexample for score monotonicity of HITS on strongly connected graphs.

Finally, the counterexample in Figure 12 shows that HITS is not score monotone on strongly connected graphs: before adding the $\operatorname{arc} x \rightarrow y$, the score of $y$ is zero, and it remains zero. $^{6}$

We are left with two open problem: is the dominant left eigenvector rank or score monotone on strongly connected graphs? We can give a direct positive answer for the first question, using Theorem 2.1 from (Elsner et al., 1982), which implies that adding an arc $x \rightarrow y$ to a strongly connected graph with dominant left eigenvector $\boldsymbol{r}$ strictly increases all ratios $r_{y} / r_{z}$ for all $z \neq y$. An immediate consequence is strict rank monotonicity.

However, while the question about rank monotonicity can be posed unambiguously even if the score is defined only up to a multiplicative constant, to prove score monotonicity we need to establish a canonical dominant left eigenvector. We choose $\ell_{1}$-normalization, as in the case of Markov chains: under this assumption, once again the result from (Elsner et al., 1982) proves score monotonicity of the dominant left eigenvector for strongly connected graphs.

\section{Conclusions and future work}

All our results are summarized in Table 1, where we distilled them into simple yes/no answers to the question: does a given centrality measure satisfy the axioms? For sake of completeness, the table contains also results proved in (Boldi \& Vigna, 2014).

Once again, only harmonic centrality satisfies all axioms. Betweenness exhibits possibly the worst behavior, failing to satisfy all axioms, even on strongly connected graphs. This is in line with results in (Boldi \& Vigna, 2014) based on information retrieval, in which betweenness performed in a way that is essentially indistinguishable from a random score.

It is interesting to note that indegree satisfies all axioms, except for size. Indeed, indegree turns out to be a surprisingly strong baseline in a number of contexts.

Our results provide further evidence that harmonic centrality should always be used instead of closeness. Closeness does not satisfy strict rank monotonicity, for reasons that are related to the fact that it does not satisfy the density axiom (Boldi \& Vigna, 2014). So, even on strongly connected graphs it is more advisable to use harmonic centrality. Our

6 Note that the counterexamples in Figure 11 and Figure 12 are built on the same principles as that in Figure 10, and nodes with the same names play the same role. Their rather awkward construction is due to the fact that an arc in Figure 10 must be replaced with a common predecessor in the other two examples, as in HITS rank propagates by zig-zag paths, that is, by common predecessors (remember that HITS is the left dominant eigenvector of $A^{T} A$ ). After that, a few additional wellplaced arcs are necessary to get a strongly connected graph. 
Table 1. Overall results from (Boldi \& Vigna, 2014) and this paper. "yes*” means that the strict version of rank monotonicity is satisfied. The results about rank monotonicity for Katz and PageRank hold for all parameters under the hypothesis that all the scores are nonzero. Score monotonicity of the dominant left eigenvector for strongly connected graphs assumes $\ell_{1}$-normalization of the scores. The centrality indices are listed based on the number of axioms they meet.

\begin{tabular}{|c|c|c|c|c|c|c|}
\hline \multirow[b]{3}{*}{ Centrality } & \multicolumn{4}{|c|}{ Monotonicity } & \multirow{2}{*}{\multicolumn{2}{|c|}{ Other axioms }} \\
\hline & \multicolumn{2}{|c|}{ General } & \multicolumn{2}{|c|}{ Strongly connected } & & \\
\hline & Score & Rank & Score & Rank & Size & Density \\
\hline Harmonic & yes & yes* & yes & yes* & yes & yes \\
\hline Degree & yes & yes* & yes & yes* & only $k$ & yes \\
\hline Katz & yes & yes* & yes & yes* & only $k$ & yes \\
\hline PageRank & yes & yes* & yes & yes* & no & yes \\
\hline Dominant & no & no & yes & yes* & only $k$ & yes \\
\hline Seeley & no & no & yes & yes & no & yes \\
\hline Lin & no & no & yes & yes & only $k$ & no \\
\hline Closeness & no & no & yes & yes & no & no \\
\hline HITS & no & no & no & no & only $k$ & yes \\
\hline SALSA & no & no & no & no & no & yes \\
\hline Betweenness & no & no & no & no & only $p$ & no \\
\hline
\end{tabular}

counterexamples show, for instance, that if you have two persons $P$ and $Q$ on Twitter with the same closeness centrality, adding a follower just to $P$ might not make it more important than $Q-$ a very counterintuitive behavior.

An interesting open problem concerns a different normalization for the dominant left eigenvector obtained using eigenprojectors. Since the eigenspace associated to the dominant eigenvalue $\lambda_{0}$ has dimension one, there is an associated eigenprojection $E_{\lambda_{0}}$. One could define the canonical dominant left eigenvector as the vector $1 E_{\lambda_{0}}$. This is in analogy with the theory of Markov chains, where the eigenprojector of the transition matrix maps initial distributions to limit stationary distributions (modulo convergence problems due to periodicities).

Given a dominant left eigenvector $\ell$ and a dominant right eigenvector $\boldsymbol{r}$ normalized so that $\ell \cdot \boldsymbol{r}=1$, the eigenprojector is given by

$$
E_{\lambda_{0}}=\boldsymbol{r}^{T} \boldsymbol{\ell},
$$

yielding

$$
1 E_{\lambda_{0}}=1 r^{T} \ell=\sum_{i} r_{i} \ell=\|r\|_{1} \ell .
$$

Note that in the case of stochastic matrices the resulting canonical eigenvector is simply the unique stationary distribution multiplied by $n$. Proving that $1 E_{\lambda_{0}}$ is score monotone is left as an open problem. 


\section{References}

Altman, Alon, \& Tennenholtz, Moshe. (2008). Axiomatic foundations for ranking systems. Journal of artificial intelligence research, 31(1), 473-495.

Anthonisse, Jacob M. (1971). The rush in a directed graph. Tech. rept. BN 9/71. Mathematical Centre, Amsterdam.

Bavelas, A. (1948). A mathematical model for group structures. Human organization, 7, 16-30.

Bavelas, A., Barrett, D., \& Association, American Management. (1951). An experimental approach to organizational communication. Publications (Massachusetts Institute of Technology. Dept. of Economics and Social Science).: Industrial Relations. American Management Association.

Beauchamp, Murray A. (1965). An improved index of centrality. Behavioral science, 10(2), 161163.

Berge, Claude. (1958). Théorie des graphes et ses applications. Paris, France: Dunod.

Berman, Abraham, \& Plemmons, Robert J. (1994). Nonnegative matrices in the mathematical sciences. Classics in Applied Mathematics. SIAM.

Boldi, Paolo, \& Vigna, Sebastiano. (2014). Axioms for centrality. Internet math., 10(3-4), 222-262.

Bonacich, Phillip. (1991). Simultaneous group and individual centralities. Social networks, 13(2), 155-168.

Brandes, Ulrik, Kosub, Sven, \& Nick, Bobo. (2012). Was messen Zentralitätsindizes? Pages 33-52 of: Hennig, Marina, \& Stegbauer, Christian (eds), Die integration von theorie und methode in der netzwerkforschung. VS Verlag für Sozialwissenschaften.

Chien, Steve, Dwork, Cynthia, Kumar, Ravi, Simon, Daniel R., \& Sivakumar, D. (2004). Link evolution: Analysis and algorithms. Internet math., 1(3), 277-304.

Cohn, B.S., \& Marriott, M. (1958). Networks and centres of integration in Indian civilization. Journal of social research, 1, 1-9.

Dequiedt, Vianney, \& Zenou, Yves. (2014). Local and consistent centrality measures in networks. Tech. rept. 2014:4. Stockholm University, Department of Economics.

Elsner, Ludwig F., Johnson, C.R., \& Neumann, Michael M. (1982). On the effect of the perturbation of a nonnegative matrix on its Perron eigenvector. Czech. math. j., 32(1), 99-109.

Fishburn, Peter C. (1982). Monotonicity paradoxes in the theory of elections. Discrete applied mathematics, 4(2), 119 - 134.

Freeman, L. (1979). Centrality in social networks: Conceptual clarification. Social networks, 1(3), 215-239.

Freeman, Linton C. (1977). A set of measures of centrality based on betweenness. Sociometry, 40(1), 35-41.

Garg, Manuj. (2009). Axiomatic foundations of centrality in networks. Social science research network.

Hubbell, Charles H. (1965). An input-output approach to clique identification. Sociometry, 28(4), 377-399.

Katz, Leo. (1953). A new status index derived from sociometric analysis. Psychometrika, 18(1), 39-43.

Kitti, Mitri. (2016). Axioms for centrality scoring with principal eigenvectors. Social choice and welfare, 46(3), 639-653.

Kleinberg, Jon M. (1999). Authoritative sources in a hyperlinked environment. Journal of the ACM, 46(5), 604-632.

Leavitt, H. J. (1951). Some effects of certain communication patterns on group performance. $J$. abnorm. psychol., 46(1), 38-50.

Lempel, Ronny, \& Moran, Shlomo. (2001). SALSA: the stochastic approach for link-structure analysis. ACM trans. inf. syst., 19(2), 131-160. 
Lin, Nan. (1976). Foundations of social research. New York: McGraw-Hill.

Mackenzie, Kenneth. (1966). Structural centrality in communications networks. Psychometrika, 31(1), 17-25.

McDonald, J.J., Neumann, M., Schneider, H., \& Tsatsomeros, M.J. (1995). Inverse $M$-matrix inequalities and generalized ultrametric matrices. Linear algebra and its applications, 220, 321 341.

Nieminen, U.J. (1973). On the centrality in a directed graph. Social science research, 2(4), 371-378.

Page, Lawrence, Brin, Sergey, Motwani, Rajeev, \& Winograd, Terry. (1998). The PageRank citation ranking: Bringing order to the web. Tech. rept. SIDL-WP-1999-0120. Stanford Digital Library Technologies Project, Stanford University.

Palacios-Huerta, Ignacio, \& Volij, Oscar. (2004). The measurement of intellectual influence. Econometrica, 72(3), 963-977.

Pinski, Gabriel, \& Narin, Francis. (1976). Citation influence for journal aggregates of scientific publications: Theory, with application to the literature of physics. Information processing \& management, 12(5), 297-312.

Pitts, Forrest R. (1965). A graph theoretic approach to historical geography. The professional geographer, 17(5), 15-20.

Sabidussi, G. (1966). The centrality index of a graph. Psychometrika, 31(4), 581-603.

Seeley, John R. (1949). The net of reciprocal influence: A problem in treating sociometric data. Canadian journal of psychology, 3(4), 234-240.

Wei, Teh-Hsing. (1952). The algebraic foundations of ranking theory. Ph.D. thesis, University of Cambridge.

Willoughby, Ralph A. (1977). The inverse M-matrix problem. Linear algebra and its applications, 18(1), 75-94. 